\title{
Bat Algorithm: Literature Review and Applications
}

\author{
Xin-She Yang \\ School of Science and Technology, Middlesex University, \\ The Burroughs, London NW4 4BT, United Kingdom. \\ Reference to this article: Xin-She Yang, Bat algorithm: literature review and \\ applications, Int. J. Bio-Inspired Computation, Vol. 5, No. 3, pp. 141-149 (2013). \\ DOI: 10.1504/IJBIC.2013.055093
}

\begin{abstract}
Bat algorithm (BA) is a bio-inspired algorithm developed by Yang in 2010 and BA has been found to be very efficient. As a result, the literature has expanded significantly in the last 3 years. This paper provides a timely review of the bat algorithm and its new variants. A wide range of diverse applications and case studies are also reviewed and summarized briefly here. Further research topics are also discussed.
\end{abstract}

\section{Introduction}

Modern optimisation algorithms are often nature-inspired, typically based on swarm intelligence. The ways for inspiration are diverse and consequently algorithms can be many different types. However, all these algorithms tend to use some specific characteristics for formulating the key updating formulae. For example, genetic algorithms were inspired by Darwinian evolution characteristics of biological systems, and genetic operators such as crossover and mutation and selection of the fittest are used. Solutions in genetic algorithms are represented as chromosomes or binary/real strings. On the other hand, particle swarm optimisation (PSO) was based on the swarming behaviour of birds and fish, and this multi-agent system may have emergent characteristics of swarm or group intelligence (Kennedy and Eberhart, 1995). Many variants of PSO and improvements exist in the literature, and many new metaheuristic algorithms have been developed (Cui, 2009; Yang, 2010; Yang and Deb, 2010; Yang et al., 2011; Yang et al., 2013).

Algorithms such as genetic algorithms and PSO can be very useful, but they still have some drawbacks in dealing with multimodal optimization problems. One major improvement is the firefly algorithm (FA) which was based on the flashing characteristics of tropical fireflies (Yang, 2008). The attraction behaviour, light intensity coding, and distance dependence provides a surprising capability to enable firefly algorithm to handle nonlinear, multimodal optimization problems efficiently. Furthermore, cuckoo search (CS) was based on the brooding behaviour of some cuckoo species (Yang and Deb, 2009; Gandomi et al, 2013) which was combined with Lévy flights. The CS algorithm is efficient because it has very good convergence behaviour that can be proved using Markovian probability theory. Other methods such as eagle strategy are also very effective (Yang and Deb, 2010; Gandomi et al, 2012).

As a novel feature, bat algorithm (BA) was based on the echolocation features of microbats (Yang, 2010), and BA uses a frequency-tuning technique to increase the diversity of the solutions in the population, while at the same, it uses the automatic zooming to try to balance exploration and exploitation during the search process by 
mimicking the variations of pulse emission rates and loudness of bats when searching for prey. As a result, it proves to be very efficient with a typical quick start. Obviously, there is room for improvement. Therefore, this paper intends to review the latest developments of the bat algorithm. The paper is organized as follows: Section 2 introduces the basic behaviour of echolocation and the standard formulation of the bat algorithm. Section 3 provides a brief description of the variants of BA, and Section 4 highlights the diverse applications of bat algorithm and its variants. Finally, Section 5 provides some discussions and topics for further research.

\section{The Standard Bat Algorithm}

The standard bat algorithm was based on the echolocation or bio-sonar characteristics of microbats. Before we outline the details of the algorithm, let us briefly introduce the echolocation.

\subsection{Echolocation of Microbats}

There are about 1000 different species of bats ( Colin, 2000). Their sizes can vary widely, ranging from the tiny bumblebee bat of about 1.5 to 2 grams to the giant bats with wingspan of about $2 \mathrm{~m}$ and may weight up to about $1 \mathrm{~kg}$. Most bats uses echolocation to a certain degree; among all the species, microbats use echolocation extensively, while megabats do not.

Microbats typically use a type of sonar, called, echolocation, to detect prey, avoid obstacles, and locate their roosting crevices in the dark. They can emit a very loud sound pulse and listen for the echo that bounces back from the surrounding objects (Richardson, 2008). Their pulses vary in properties and can be correlated with their hunting strategies, depending on the species. Most bats use short, frequency-modulated signals to sweep through about an octave, and each pulse lasts a few thousandths of a second (up to about 8 to $10 \mathrm{~ms}$ ) in the frequency range of $25 \mathrm{kHz}$ to $150 \mathrm{kHz}$. Typically, microbats can emit about 10 to 20 such sound bursts every second, and the rate of pulse emission can be sped up to about 200 pulses per second when homing on their prey. Since the speed of sound in air is about $v=340 \mathrm{~m} / \mathrm{s}$, the wavelength $\lambda$ of the ultrasonic sound bursts with a constant frequency $f$ is given by $\lambda=v / f$, which is in the range of $2 \mathrm{~mm}$ to $14 \mathrm{~mm}$ for the typical frequency range from $25 \mathrm{kHz}$ to $150 \mathrm{kHz}$. Interestingly, these wavelengths are in the same order of their prey sizes.

Though in reality microbats can also use time delay between their ears and loudness variations to sense three-dimensional surroundings, we are mainly interested in some features of the echolocation so that we can some link them with the objective function of an optimization problem, which makes it possible to formulate a smart, bat algorithm.

\subsection{Bat Algorithm}

Based on the above description and characteristics of bat echolocation, Xin-She Yang (2010) developed the bat algorithm with the following three idealised rules:

1. All bats use echolocation to sense distance, and they also 'know' the difference between food/prey and background barriers in some magical way;

2. Bats fly randomly with velocity $\boldsymbol{v}_{i}$ at position $\boldsymbol{x}_{i}$ with a frequency $f_{\min }$, varying wavelength $\lambda$ and loudness $A_{0}$ to search for prey. They can automatically adjust the wavelength (or frequency) of their emitted pulses and adjust the rate of pulse emission $r \in[0,1]$, depending on the proximity of their target;

3. Although the loudness can vary in many ways, we assume that the loudness varies from a large (positive) $A_{0}$ to a minimum constant value $A_{\min }$. 
For simplicity, we do not use ray tracing in this algorithm, though it can form an interesting feature for further extension. In general, ray tracing can be computational extensive, but it can be a very useful feature for computational geometry and other applications. Furthermore, a given frequency is intrinsically linked to a wavelength. For example, a frequency range of $[20 \mathrm{kHz}, 500 \mathrm{kHz}]$ corresponds to a range of wavelengths from $0.7 \mathrm{~mm}$ to $17 \mathrm{~mm}$ in the air. Therefore, we can describe the change either in terms of frequency $f$ or wavelength $\lambda$ to suit different applications, depending on the ease of implementation and other factors.

\subsection{Bat Motion}

Each bat is associated with a velocity $\boldsymbol{v}_{i}^{t}$ and a location $\boldsymbol{x}_{i}^{t}$, at iteration $t$, in a $d$ dimensional search or solution space. Among all the bats, there exists a current best solution $\boldsymbol{x}_{*}$. Therefore, the above three rules can be translated into the updating equations for $\boldsymbol{x}_{i}^{t}$ and velocities $\boldsymbol{v}_{i}^{t}$ :

$$
\begin{gathered}
f_{i}=f_{\min }+\left(f_{\max }-f_{\min }\right) \beta, \\
\boldsymbol{v}_{i}^{t}=\boldsymbol{v}_{i}^{t-1}+\left(\boldsymbol{x}_{i}^{t-1}-\boldsymbol{x}_{*}\right) f_{i}, \\
\boldsymbol{x}_{i}^{t}=\boldsymbol{x}_{i}^{t-1}+\boldsymbol{v}_{i}^{t},
\end{gathered}
$$

where $\beta \in[0,1]$ is a random vector drawn from a uniform distribution.

As mentioned earlier, we can either use wavelengths or frequencies for implementation, we will use $f_{\min }=0$ and $f_{\max }=O(1)$, depending on the domain size of the problem of interest. Initially, each bat is randomly assigned a frequency which is drawn uniformly from $\left[f_{\min }, f_{\max }\right]$. For this reason, bat algorithm can be considered as a frequency-tuning algorithm to provide a balanced combination of exploration and exploitation. The loudness and pulse emission rates essentially provide a mechanism for automatic control and auto zooming into the region with promising solutions.

\subsection{Variations of Loudness and Pulse Rates}

In order to provide an effective mechanism to control the exploration and exploitation and switch to exploitation stage when necessary, we have to vary the loudness $A_{i}$ and the rate $r_{i}$ of pulse emission during the iterations. Since the loudness usually decreases once a bat has found its prey, while the rate of pulse emission increases, the loudness can be chosen as any value of convenience, between $A_{\min }$ and $A_{\max }$, assuming $A_{\min }=0$ means that a bat has just found the prey and temporarily stop emitting any sound. With these assumptions, we have

$$
A_{i}^{t+1}=\alpha A_{i}^{t}, \quad r_{i}^{t+1}=r_{i}^{0}[1-\exp (-\gamma t)],
$$

where $\alpha$ and $\gamma$ are constants. In essence, here $\alpha$ is similar to the cooling factor of a cooling schedule in simulated annealing. For any $0<\alpha<1$ and $\gamma>0$, we have

$$
A_{i}^{t} \rightarrow 0, \quad r_{i}^{t} \rightarrow r_{i}^{0}, \text { as } t \rightarrow \infty .
$$

In the simplest case, we can use $\alpha=\gamma$, and we have used $\alpha=\gamma=0.9$ to 0.98 in our simulations.

\section{Variants of Bat Algorithm}

The standard bat algorithm has many advantages, and one of the key advantages is that it can provide very quick convergence at a very initial stage by switching from exploration to exploitation. This makes it an efficient algorithm for applications such 
as classifications and others when a quick solution is needed. However, if we allow the algorithm to switch to exploitation stage too quickly by varying $A$ and $r$ too quickly, it may lead to stagnation after some initial stage. In order to improve the performance, many methods and strategies have been attempted to increase the diversity of the

solution and thus to enhance the performance, which produced a few good variants of bat algorithm.

From a quick literature survey, we found the following bat algorithm variants:

- Fuzzy Logic Bat Algorithm (FLBA): Khan et al. (2011) presented a variant by introducing fuzzy logic into the bat algorithm, they called their variant fuzzy bat algorithm.

- Multiobjective bat algorithm (MOBA): Yang (2011) extended BA to deal with multiobjective optimization, which has demonstrated its effectiveness for solving a few design benchmarks in engineering.

- K-Means Bat Algorithm (KMBA): Komarasamy and Wahi (2012) presented a combination of K-means and bat algorithm (KMBA) for efficient clustering.

- Chaotic Bat Algorithm (CBA): Lin et al. (2012) presented a chaotic bat algorithm using Lévy flights and chaotic maps to carry out parameter estimation in dynamic biological systems.

- Binary bat algorithm (BBA): Nakamura et al. (2012) developed a discrete version of bat algorithm to solve classifications and feature selection problems.

- Differential Operator and Lévy flights Bat Algorithm (DLBA): Xie et al. (2013) presented a variant of bat algorithm using differential operator and Lévy flights to solve function optimization problems.

- Improved bat algorithm (IBA): Jamil et al. (2013) extended the bat algorithm with a good combination of Lévy flights and subtle variations of loudness and pulse emission rates. They tested the IBA versus over 70 different test functions and proved to be very efficient.

There are other improvements and variants of bat algorithm. For example, Zhang and Wang (2012) used mutation to enhance the diversity of solutions and then used for image matching. In addition, Wang and Guo (2013) hybridized bat algorithm with harmony search and have produced a hybrid bat algorithm for numerical optimization of function benchmarks.

On the other hand, Fister Jr et al. (2013) developed a hybrid bat algorithm using differential evolution as a local search part of bat algorithm, while Fister et al. (2013) incorporate quaternions into bat algorithm and presented a quaternion bat algorithm (QBA) for computational geometry and large-scale optimization problems with extensive rotations. It can be expect that more variants are still under active research.

\section{Applications of Bat Algorithm}

The standard bat algorithm and its many variants mean that the applications are also very diverse. In fact, since the original bat algorithm has been developed (Yang, 2010), Bat algorithms have been applied in almost every area of optimization, classifications, image processing, feature selection, scheduling, data mining and others. In the rest of the paper, we will briefly highlight some of the applications (Yang, 2010;Parpinelli and Lopes, 2011; Yang et al., 2012a; Yang, 2012; Yang, 2013; Gandomi et al., 2013).

\subsection{Continuous Optimization}

Among the first set of applications of bat algorithm, continuous optimization in the context of engineering design optimization has been extensively studied, which demonstrated that BA can deal with highly nonlinear problem efficiently and can find the 
optimal solutions accurately (Yang, 2010; Yang and Gandomi, 2012; Yang, 2012; Yang et al., 2012a). Case studies include pressure vessel design, car side design, spring and beam design, truss systems, tower and tall building design and others. Tsai et al. (2011) solved numerical optimization problems using bat algorithm.

In addition, Bora et al. (2012) optimized the brushless DC wheel motors using bat algorithm with superior results. BA can also handle multiobjective problems effectively (Yang, 2011).

\subsection{Combinatorial Optimization and Scheduling}

From computational complexity point of view, continuous optimization problems can be considered as easy, though it may be still very challenging to solve. However, combinatorial problems can be really hard, often non-deterministic polynomial time hard (NP-hard). Ramesh et al. (2013) presented a detailed study of combined economic load and emission dispatch problems using bat algorithm. They compared bat algorithm with ant colony algorithm $(\mathrm{ABC})$, hybrid genetic algorithm and other methods, and they concluded that bat algorithm is easy to implement and much superior to the algorithms in terms of accuracy and efficiency.

Musikapun and Pongcharoen (2012) solved multi-stage, multi-machine, multi-product scheduling problems using bat algorithm, and they solved a class of non-deterministic polynomial time (NP) hard problems with a detailed parametric study. They also implied that that the performance can be further improved by about $8.4 \%$ using optimal set of parameters.

\subsection{Inverse Problems and Parameter Estimation}

Yang et al. (2012b) use the bat algorithm to study topological shape optimization in microelectronic applications so that materials of different thermal properties can be placed in such a way that the heat transfer is most efficient under stringent constraints. It can also be applied to carry out parameter estimation as an inverse problem. If an inverse problem can be properly formulated, then bat algorithm can provide better results than least-squares methods and regularization methods.

Lin et al. (2012) presented a chaotic Lévy flight bat algorithm to estimate parameters in nonlinear dynamic biological systems, which proved the effectiveness of the proposed algorithm.

\subsection{Classifications, Clustering and Data Mining}

Komarasamy and Wahi (2012) studied K-means clustering using bat algorithm and they concluded that the combination of both K-means and BA can achieve higher efficiency and thus performs better than other algorithms.

Khan et al. (2011) presented a study of a clustering problem for office workplaces using a fuzzy bat algorithm. Khan and Sahari (2012a) also presented a comparison study of bat algorithm with PSO, GA, and other algorithms in the context for e-learning, and thus suggested that bat algorithm has clearly some advantages over other algorithms. Then, they (Khan and Sahari, 2012b) also presented a study of clustering problems using bat algorithm and its extension as a bi-sonar optimization variant with good results.

On the other hand, Mishra et al. (2012) used bat algorithm to classify microarray data, while Natarajan et al. (2012) presented a comparison study of cuckoo search and bat algorithm for Bloom filter optimization. Damodaram and Valarmathi (2012) studied phishing website detection using modified bat algorithm and achieved very good results. 
Marichelvam and Prabaharan (2012) used bat algorithm to study hybrid flow shop scheduling problems so as to minimize the makespan and mean flow time. Their results suggested that BA is an efficient approach for solving hybrid flow shop scheduling problems. Faritha Banu and Chandrasekar (2013) used a modified bat algorithm to record deduplication as an optimization approach and data compression technique. Their study suggest that the modified bat algorithm can perform better than genetic programming.

\subsection{Image Processing}

Abdel-Rahman et al. (2012) presented a study for full body human pose estimation using bat algorithm, and they concluded that BA performs better than particle swarm optimization (PSO), particle filter (PF) and annealed particle filter (APF).

Du and Liu (2012) presented a variant of bat algorithm with mutation for image matching, and they indicated that their bat-based model is more effective and feasible in imagine matching than other models such as differential evolution and genetic algorithms.

\subsection{Fuzzy Logic and Other Applications}

Reddy and Manoj (2012) presented a study of optimal capacitor placement for loss reduction in distribution systems using bat algorithm. It combines with fuzzy logic to find optimal capacitor sizes so as to minimize the losses. Their results suggested that the real power loss can be reduced significantly.

Furthermore, Lemma et al. (2011) used fuzzy systems and bat algorithm for exergy modelling, and later Tamiru and Hashim (2013) applied bat algorithm to study fuzzy systems and to model exergy changes in a gas turbine.

At the time of writing when we searched the Google scholar and other databases, we found other papers on bat algorithm that were either just accepted or conference presentations. However, there is not enough detail to be included in this review. In fact, as the literature is expanding, more and more papers on bat algorithm are emerging, a further timely review will be needed within the next two years.

\section{Discussions and Conclusions}

Likely many metaheuristic algorithms, bat algorithm has the advantage of simplicity and flexibility. BA is easy to implement, and such a simple algorithm can be very flexible to solve a wide range of problems as we have seen in the above review.

\subsection{Why Bat Algorithm is Efficient}

A natural question is: why bat algorithm is so efficient? There are many reasons for the success of bat-based algorithms. By analysing the key features and updating equations, we can summarize the following three key points/features:

- Frequency tuning: BA uses echolocation and frequency tuning to solve problems. Though echolocation is not directly used to mimic the true function in reality, frequency variations are used. This capability can provide some functionality that may be similar to the key feature used in particle swarm optimization and harmony search. Therefore, BA possess the advantages of other swarm-intelligence-based algorithms.

- Automatic zooming: BA has a distinct advantage over other metaheuristic algorithms. That is, BA has a capability of automatically zooming into a region where promising solutions have been found. This zooming is accompanied by the 
automatic switch from explorative moves to local intensive exploitation. As a result, BA has a quick convergence rate, at least at early stages of the iterations, compared with other algorithms.

- Parameter control: Many metaheuristic algorithms used fixed parameters by using some, pre-tuned algorithm-dependent parameters. In contrast, BA uses parameter control, which can vary the values of parameters $(A$ and $r)$ as the iterations proceed. This provides a way to automatically switch from exploration to exploitation when the optimal solution is approaching. This gives another advantages of BA over other metaheuristic algorithms.

In addition, preliminary theoretical analysis by Huang et al.(2013) suggested that BA has guaranteed global convergence properties under the right condition, and BA can also solve large-scale problems effectively.

\subsection{Further Research Topics}

However, there are still some important issues that require more research. These key issues are: parameter-tuning, parameter control and speedup of convergence.

Firstly, parameter-tuning is important for any metaheuristic algorithm to work properly. In almost all cases, the performance of an algorithm is largely dependent on the parameters of the algorithm. To find the best parameter settings, detailed parametric studies have to be carried out. It is not known yet if there is a method to automatically tune parameters for an algorithm to achieve the optimal performance for a given set of problems. This should be an important topic for further research.

Secondly, associated with the parameter tuning, there is an important issue of parameter control. In many algorithms, the parameter settings are fixed, and these settings will not vary during the iterations. It could be advantageous and sometime necessary to vary the values of algorithm-dependent parameters during the iterative search process. How to vary or control these parameters is another, higher level, optimization problem, which needs further studies. For bat algorithm, we have introduced the basic parameter control strategy, there is still room for improvement. An open question is that: what is the best control strategy so as to switch from exploration to exploitation at the right time?

Finally, even though the bat algorithm and other algorithms are efficient, it is still possible to improve and enhance their performance further. However, how to speed up the convergence of an algorithm is still a very challenging question. It is hoped this this paper can inspire more research in the near future. Future research should focus on the theoretical understanding of metaheuristic algorithms and large-scale problems in real-world applications.

\section{References}

[1] Abdel-Rahman, E. M., Ahmad, A. R., Akhtar, S., (2012). A metaheurisic batinspired algorithm for full body human pose estimation, in: Ninth Conference on Computer and Robot Vision, pp. 369-375.

[2] Bora, T. C., Coelho, L. S., Lebensztajn, L., (2012). Bat-inspired optimization approach for the brushless DC wheel motor problem, IEEE Trans. Magnetics, Vol. 48, No. 2, 947-950 (2012).

[3] Colin, T., (2000). The Varienty of Life. Oxford University Press, Oxford.

[4] Cui, Z. H., and Cai, X. J. (2009). Integral particle swarm optimisation with dispersed accelerator information, Fundam. Inform., Vol. 95, 427-447. 
[5] Damodaram, R., Valarmathi, M. L., (2012). Phishing website detection and optimization using modified bat algorithm, Int. J. Engineering Research and Applications, Vol. 2, No. 1, pp. 870-876.

[6] Du, Z. Y., Liu B., (2012). Image matching using a bat algorithm with mutation, Applied Mechanics and Materials, Vol. 203, No. 1, pp. 88-93.

[7] Faritha Banu, A., Chandrasekar, C., (2012). An optimized appraoch of modified bat algorithm to record deduplication, Int. Journal of Computer Applications, Vol. 62, No. 1, pp. 10-15.

[8] Fister Jr., I., Fister, D., and Yang, X. S., (2013). A hybrid bat algorithm, Elekrotehniški Vestnik (English Edition), (2013, submitted).

[9] Fister, I., Fister Jr., I., Yang, X. S., and Brest, J., (2013). On the representation of individual s using quaternions in swarm intelligence and evolutionary computation, IEEE Trans. Evol. Computation, (2013, submitted).

[10] Gandomi, A. H., Yang, X. S., Talatahari, S., and Deb, S., (2012). Coupled eagle strategy and differential evolution for unconstrained and constrained global optimization, Computers 83 Mathematics with Applications, vol. 63, no. 1, pp. 191-200.

[11] Gandomi, A. H., Yang, X. S., Alavi, A. H., Talatahari , S. (2013). Bat algorithm for constrained optimization tasks, Neural Computing and Applications, http://link.springer.com/article/10.1007

[12] Huang, G. Q., Zhao, W. J., and Lu, Q. Q., (2013). Bat algorithm with global convergence for solving large-scale optimization problem, Application Research of Computers, vol. 30, no. 3, 1-10 (in Chinese).

[13] Jamil, M., Zepernic, H.-J., and Yang, X. S., (2013). Improved bat algorithm for global optimization, Applied Soft Computing, (2013, submitted).

[14] Khan, K., Nikov, A., Sahai A., (2011). A fuzzy bat clustering method for ergonomic screening of office workplaces, S3T 2011, Advances in Intelligent and Soft Computing, 2011, Volume 101/2011, pp. 59-66.

[15] Khan, K., and Sahai, A., (2012a). A comparison of BA, GA, PSO, BP and LM for training feed forward neural networks in e-learning context, Int. J. Intelligent Systems and Applications (IJISA), Vol. 4, No. 7, pp. 23-29.

[16] Khan, K., and Sahai, A., (2012b). A fuzzy c-means bi-sonar-based metaheuristic optimization algorithm, INt. J. of Interactive Multimedia and Artificial Intelligence, Vol. 1, no. 7, pp. 26-32.

[17] Komarasamy, G., and Wahi, A., (2012). An optimized K-means clustering techniqueusing bat algorithm, European J. Scientific Research, Vol. 84, No. 2, pp. 263-273.

[18] Lemma, T. A., Bin Mohd Hashim, F., (2011). Use of fuzzy systems and bat algorithm for exergy modelling in a gas turbine generator, IEEE Colloquium on Humanities, Science and Engineering (CHUSER'2011), 5-6 Dec. 2011, pp. 305-310.

[19] Lin, J. H., Chou,C. W., Yang, C. H.,Tsai, H. L., (2012). A chaotic Levy flight bat algorithm for parameter estimation in nonlinear dynamic biological systems, J.Computer and Information Technology, Vol. 2, No. 2, pp. 56-63.

[20] Marichelvam, M. K., and Prabaharam, T., (2012). A bat algorithm for realistic hybrid flowshop schedulihng problems to minimize makespan and mean flow time, ICTACT Journal on Soft Computing, Vol. 3, No. 1, pp. 428-433.

[21] Mishra, S., Shaw, K., Mishra, D., (2012). A new meta-heuristic bat inspired classification approach for microarray data, Procedia Technology, Vol. 4, pp. 802806. 
[22] Musikapun, P., Pongcharoen, P., Solving multi-stage multi-machine multiproduct scheduling problem using bat aglorithm, (2012). 2nd International Conference on Management and Artificial Intelligence (IPEDR),Vol. 35, IACSIT Press, Singapore, pp. 98-102.

[23] Nakamura, R. Y. M., Pereira, L. A. M., Costa, K. A., Rodrigues, D., Papa, J. P., Yang, X. S., (2012). BBA: A binary bat algorithm for feature selection, in: 25th SIBGRAPI Conference on Graphics, Patterns and Images (SIBGRAPI), 22-25 Aug. 2012, IEEE Publication, pp. 291-297.

[24] Natarajan, A., Subramanian, S., Premalatha, K., (2012). A comparative study of cuckoo search and bat algorithm for Bloom filter optimisation in spam filtering, Int. J. Bio-Inspired Computation, Vol. 4, No. 2, pp. 89-99.

[25] Parpinelli, R. S., and Lopes, H. S., (2011). New inspirations in swarm intelligence: a survey, Int. J. Bio-Inspired Computation, Vol. 3, No. 1, pp. 1-16.

[26] Ramesh, B., Mohan, V. C. J., Reddy, V. C. V., (2013). Application of bat algorithm for combined economic load and emission dispatch, Int. J. of Electricl Engineering and Telecommunications, Vol. 2, No. 1, pp. 1-9.

[27] Reddy, V. U., Manoj, A., (2012). Optimal capacitor placement for loss reduction in distribution systems using bat algorithm, IOSR Journal of Engineering, Vol. 2, No. 10, pp. 23-27.

[28] Richardson, P., (2008). Bats. Natural History Museum, London.

[29] Tamiru, A. L., Hashim, F. M., (2013). Application of bat algorithm and fuzzy systems to model exergy changes in a gas turbine, in: Artificial Intelligence, Evolutionary Computing and Metaheuristics (Eds. X. S. Yang), Studies in Computational Intelligence, Vol. 427, Springer, Heidelberg, pp. 685-719.

[30] Tsai, P. W., Pan, J. S., Liao, B. Y., Tsai, M. J., Istanda, V., (2011). Bat algorithm inspired algorithm for solving numerical optimization problems, Applied Mechanics and Materials, Vol. 148-149, pp.134-137.

[31] Wang, G. G, Guo, L. H., Duan, H., Liu, L, Wang, H. Q., (2012). A bat algorithm with mutation for UCAV path planning, Scientific World Journal, Vol. 2012, 15 pages. doi:10.1100/2012/418946 http://www.hindawi.com/journals/tswj/2012/418946/

[32] Wang, Gaige, and Guo, Lihong, (2013). A novel hybrid bat algorithm with harmony search for global numerical optimization, Journal of Applied Mathematics, (in press).

[33] Xie, J., Zhou, Y. Q., Chen,H., A novel bat algorithm based on differential operator and Lévy flights trajectory, Computational Intelligence and Neuroscience, Vol. 2013, Article ID: 453812 DOI:www.hindawi.com/journals/cin/aip/453812.pdf

[34] Yang, X. S., (2008). Nature-Inpsired Metaheursitic Algorithms, Luniver Press, Frome, UK.

[35] Yang, X. S., (2010). A New Metaheuristic Bat-Inspired Algorithm, in: Nature Inspired Cooperative Strategies for Optimization (NISCO 2010) (Eds. Cruz, C.; González, J. R.; Pelta, D. A.; Terrazas, G), Studies in Computational Intelligence Vol. 284, Springer Berlin, pp. 65-74.

[36] Yang, X. S., and Deb, S., (2010). Eagle strategy using Lévy walk and firefly algorithms for stochastic optimization, Nature Inspired Cooperative Strategies for Optimization (NICSO2010), (Eds. Cruz, C.; González, J. R.; Pelta, D. A.; Terrazas, G), Studies in Computational Intelligence Vol. 284, pp. 101-111.

[37] Yang, X. S., (2011). Bat algorithm for multi-objective optimisation, Int. J. BioInspired Computation, Vol. 3, No. 5, pp. 267-274. 
[38] Yang, X. S. and Deb, S. (2009). Cuckoo search via Lévy flights, in: Proc. of World Congress on Nature 83 Biologically Inspired Computing (NaBic 2009), IEEE Publications, USA, pp. 210-214.

[39] Yang, X. S., Deb, S., and Fong, S., (2011). Accelerated particle swarm optimization and support vector machine for business optimization and applications, in: Networked Digital Technologies 2011, Communications in Computer and Information Science, 136, pp. 53-66.

[40] Yang, X. S., (2013). Bat algorithm and cuckoo search: a tutorial, in: Artificial Intelligence, Evolutionary Computing and Metaheuristics (Eds. X. S. Yang), Studies in Computational Intelligence, Vol. 427, pp. 421-434.

[41] Yang, X. S., Cui, Z. H., Xiao, R. B., Gandomi, A. H., Karamanoglu, M., (2013). Swarm Intelligence and Bio-Inpsired Computation: Theory and Applications, Elsevier, London, (2013).

[42] Yang, X. S. and Gandomi, A. H., (2012). Bat algorithm: a novel approach for global engineering optimization, Engineering Computations, Vol. 29, No. 5, pp. 464-483.

[43] Yang, X. S., Gandomi, A. H., Talatahari, S., Alavi, A. H., (2012a). Metaheuristics in Water, Geotechnical and Transport Engineering, Elsevier, London, UK and Waltham, USA.

[44] Yang, X. S., Karamanoglu, M., Fong, S., (2012b). Bat aglorithm for topology optimization in microelectronic applications, in: IEEE Int. Conference on Future Generation Communication Technology (FGCT2012), British Computer Society, 12-14 Dec 2012, London, pp. 150-155.

[45] Yang, X. S., (2012). Metaheuristic optimization with applications: Demonstration via bat algorithm, in: Proceedings of 5th Bioinspired Optimization Methods and Their Applications (BIOMA2012) (Eds. B.Filipic and J. Silc), 24-25 May 2012, Bohini, Slovenia, pp. 23-34.

[46] Zhang, J. W., and Wang, G. G., (2012). Image matching using a bat algorithm with mutation, Applied Mechanics and Materials (Editted by Z. Y. Du and Bin Liu), Vol. 203, No. 1, pp. 88-93. 